\title{
Electron-Spin Echoes at 604 GHz Using Far Infrared Lasers
}

\author{
C. Kutter, ${ }^{1}$ H. P. Moll, ${ }^{1}$ J. van Tol, ${ }^{1}$ H. Zuckermann, ${ }^{1}$ J. C. Maan, ${ }^{2}$ and P. Wyder ${ }^{1}$ \\ ${ }^{1}$ High Magnetic Field Laboratory, Max-Planck-Institute für Festkörperforschung, Centre National de la Recherche Scientifique, \\ B.P. 166, F-38042 Grenoble CEDEX 9, France \\ ${ }^{2}$ High Magnetic Field Laboratory, Katholieke Universiteit Nijmegen, 6525 ED Nijmegen, The Netherlands.
} (Received 14 November 1994)

\begin{abstract}
We have observed electron-spin echoes at $604 \mathrm{GHz}$ in a magnetic field of $21.5 \mathrm{~T}$. This frequency and magnetic field are more than 4 times larger than frequencies and fields at which electron-spin echoes have been reported before. We have used far infrared lasers for the excitation pulses and have employed a heterodyne detection system with a Schottky diode as mixer element and a far infrared laser as local oscillator. For a relatively concentrated nitroxide free-radical sample, we show that the spin-memory time is longer at higher frequencies due to the increased spin polarization at high magnetic fields.

PACS numbers: $33.35 .+r, 76.30 . R n$
\end{abstract}

With the introduction of coherent pulsed techniques in nuclear-magnetic-resonance (NMR) and electron-spinresonance (ESR), a new field opened for the investigation of quantum dynamics of spin systems. In his first experiments, Hahn and co-workers used two- and threepulse sequences to measure the spin-memory and spinlattice relaxation times of nuclear spins [1,2].

In ESR, the spin-echo technique was introduced using the typical NMR equipment to produce the high frequency pulses (Blume 17.4 MHz [3]), and somewhat later spectrometers using microwave techniques and operating at the $X$ band $(9.5 \mathrm{GHz})$ were constructed [4-6]. Advances in micro- and millimeter wave technology in recent years created the possibility to build spectrometers operating at higher frequencies, and at present electron-spin-echo (ESE) spectrometers at $95 \mathrm{GHz}[7,8]$ and $140 \mathrm{GHz}$ [9] are in use. It will be difficult to obtain a really significant further increase in frequency with the presently available millimeter devices, which is the reason why we have chosen an optical approach with a pulsed far infrared (FIR) laser system as the high frequency source. Continuous wave (cw) high frequency EPR has been pioneered by the group of Lebedev [10]. At very high frequencies a spectrometer using Gunn diodes and optical techniques exists $(250 \mathrm{GHz})$ [11], and also a multifrequency spectrometer with a cw FIR laser as millimeter/submillimeter source $(140-2000 \mathrm{GHz})$ has proven its usefulness in $\mathrm{cw}$ EPR [12]. With our optical approach in pulsed EPR, it is, in fact, the magnetic field that becomes the limiting factor for a further increase in frequency. We have chosen $604 \mathrm{GHz}$ because of the availability of a strong laser line at this frequency and because the resonance magnetic field of $21.5 \mathrm{~T}$ for a $g$ factor of 2 is available in our laboratory.

Our ESE spectrometer extends the current frequency limit of $140 \mathrm{GHz}$ for the most modern spectrometers [9] by more than a factor of 4 , up to $604 \mathrm{GHz}$. The Zeeman splitting at this frequency corresponds to $29 \mathrm{~K}$, so that at liquid helium temperatures the spin polarization of the levels is almost complete $(99.8 \%$ at $4.2 \mathrm{~K})$, while at room temperature we still have a $5 \%$ polarization. This high polarization leads to a higher sensitivity and presents an interesting physical situation, which may lead, as we will demonstrate in this paper, to very long spin-memory times.

The high frequencies also create the possibility of studying experimentally the energy (or field) dependence of the spin-lattice $\left(T_{1}\right)$ and spin-spin $\left(T_{2}\right)$ relaxation times over a much wider range. New insight into the relaxation processes can be expected by performing $T_{1}$ and $T_{2}$ studies as a function of the magnetic field.

As in all high-field spin resonance experiments, an important advantage is the $g$-factor resolution. The 60 -fold increase in frequency with respect to the $X$ band facilitates measurement of small $g$ anisotropies and simplifies interpretation of the spectra. For powders with badly resolved $g$ anisotropy at low fields, the high frequency spectrum may reflect the orientation dependence of $g$ and creates the possibility of selecting an orientation by selecting a field position within the EPR spectrum.

Another advantage of high frequency spectrometers is that high spin systems $(S \geq 1)$ with much larger zero field splittings can be studied, since the photon energy of the high field spectrometers is much higher.

One of the requirements for pulsed EPR is the creation of intense excitation pulses. In the Hahn-type spin-echo experiments, a first $\pi / 2$ pulse, which coherently excites the spin system, is applied to the sample. After this pulse, the individual spin packets dephase due to local field inhomogeneities. A second $\pi$ pulse, applied after a delay time $\tau$, refocuses the spin packets, and another time $\tau$ later they are all in phase again, giving rise to the echo. At exact resonance, the condition for a $\pi$ pulse is given by $\pi=\gamma \Delta t B_{1}, \gamma$ being the gyromagnetic ratio, $\Delta t$ the pulse width, and $B_{1}$ the amplitude of the oscillating magnetic field. This implies that for a pulse width of $100 \mathrm{~ns}$, a magnetic field $B_{1}$ of $1.8 \mathrm{G}$ at the sample position 
is necessary, corresponding to a power density for a free traveling wave of about $30 \mathrm{~W} /\left(4 \mathrm{~mm}^{2}\right), 4 \mathrm{~mm}^{2}$ being the sample area. With less intense pulses, longer pulse lengths would be required, reducing the usefulness of the technique. The pulse power is proportional to $(\Delta t)^{-2}$, and it is clear that for pulses with a very short width $\Delta t$ (e.g., $10 \mathrm{~ns}$ ) the necessary power will be difficult to obtain.

To generate the high power, high frequency pulses, we used a pulsed FIR laser, which is a molecular gas laser optically pumped by a TEA (transversely excited atmospheric pressure) $\mathrm{CO}_{2}$ laser. Pulses with an intrinsic pulse length of about 100 ns and high pulse powers $(\approx 100 \mathrm{~W})$ can be produced, using methylfluoride, with its very intense line at $604 \mathrm{GHz}$, as FIR gas [13]. In principle, many different FIR frequencies can be generated with different FIR gases and pump lines; however, the $604 \mathrm{GHz}$ line has a comparatively high intensity. In order to create two pulses with a variable delay, two independent systems are required, because the $\mathrm{CO}_{2}$ laser cannot fire twice in quick succession and a variable optical delay with a length of 50 to $1000 \mathrm{~m}$ is not possible without changes in the power of the second pulse. By a proper adjustment of the $\mathrm{CO}_{2}$ pump lasers, the mirrors of the FIR lasers and the correct gas pressures, two single mode pulses without frequency shift and with Gaussian beam properties can be generated. For the $T_{1}$ measurements, three pulses are required: a saturation pulse, followed after a variable delay by a two-pulse echo sequence with a fixed delay between the two pulses. The first pulse is produced as described above and the two other pulses of the echo sequence are generated with a single FIR-laser system in conjunction with an optical delay line. The $\mathrm{CO}_{2}$-laser beam is split and one of the two pulses is sent into an optical delay line of $90 \mathrm{~m}$ in order to pump the FIR cavity $300 \mathrm{~ns}$ after the first pulse. In this way, a three-pulse sequence with one variable $\tau_{1}$ and one fixed delay $\tau_{2}$ can be produced as shown in Fig. 4 below. To transport the radiation to the sample, we have chosen to use oversized waveguides, hollow dielectric or metallic tubes with $10 \mathrm{~mm}$ inner diameter $(\approx 20 \lambda)$. This solution turned out to be the best compromise between low transmission losses and handling convenience. Because of the long FIR wavelength $(0.5 \mathrm{~mm})$, an all-optical solution would require many optical elements and performance would depend critically on alignment.

To increase the $B_{1}$ field on the sample in order to make $\pi$ and $\pi / 2$ pulses, and to increase the sensitivity, we use a Fabry-Pérot reflection cavity with a spherical copper mirror on the back and a 200 lines per inch copper mesh as an incouple mirror. The cavity necessitates small samples to obtain a reasonable finesse of the loaded cavity. We have used either a thin film or a very small crystal glued on the back mirror, and obtained a finesse of the loaded cavity of about 40 . The small sample volume has the advantage that the static magnetic field is still reasonably homogeneous, even in our magnet that is optimized for field strength rather than for homogeneity. Magnetic fields of $21.5 \mathrm{~T}$ can presently be generated only by resistive magnets. We have used a $10 \mathrm{MW}, 25 \mathrm{~T}$ polyhelix magnet [14] with a spatial field variation of about $5 \mathrm{G}$ over a volume of $1 \mathrm{~mm}^{3}$. The field stability due to the current in our magnets is about $10 \mathrm{G}$ at $21 \mathrm{~T}$. The fluctuations are slow on the time scale of the echo sequence and are fast compared to the repetition rate. In the present setup, this limits the resolution to about $10 \mathrm{G}$.

For the detection of the echo, we have used a heterodyne system with a Schottky diode as the mixer element, similar to the cw detection system used in submillimeter radioastronomy [15]. These detection systems offer a very good time resolution and a high sensitivity. The necessary power for the local oscillator (LO) is on the order of $\mathrm{mW}$ and can be provided by $\mathrm{cw}$ FIR lasers. We used the $611 \mathrm{GHz}$ line of fully deuterated methyliodide. The $7 \mathrm{GHz}$ intermediate frequency (IF) of the signal and the local oscillator is amplified by a low noise amplifier. A switch prevents saturation of a second amplifier. The amplified IF signal is rectified by a diode and recorded by a transient digitizer. The Schottky diode itself is protected in a passive manner by using a wire-grid polarizer as beam splitter (see Fig. 1). The radiation of the two linearly polarized excitation pulses passes the beam splitter, is partly reflected back from the cavity, and passes the beam splitter again. Since the echo is circularly polarized, half of the echo signal is reflected into the direction of the detector. This passive protection provides an isolation of only

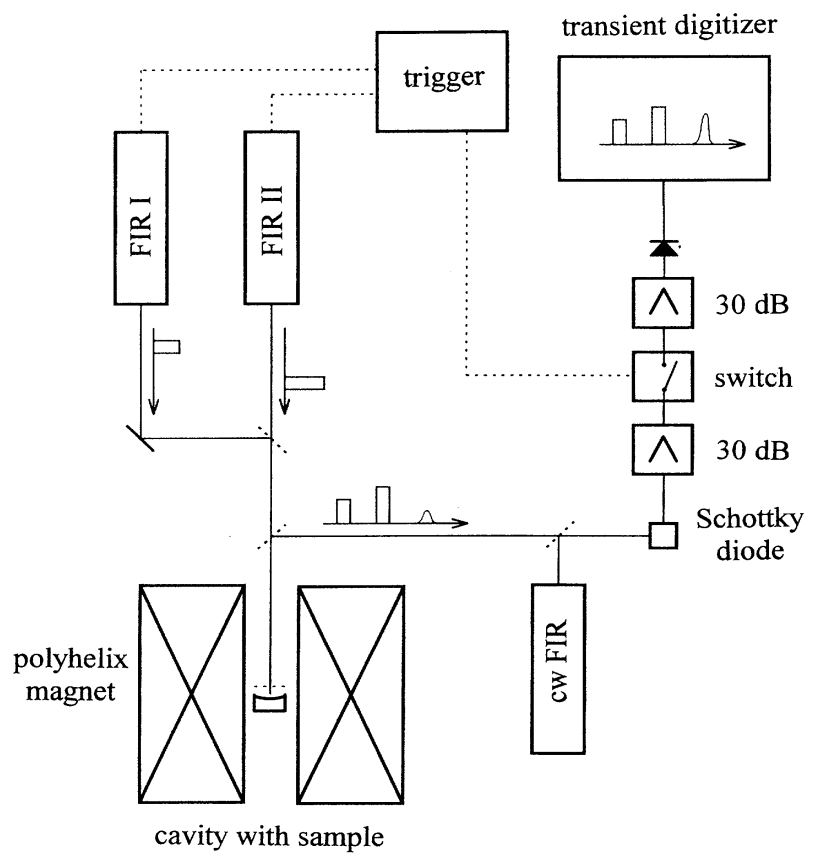

FIG. 1. Schematic setup of the $604 \mathrm{GHz}$ spectrometer showing the arrangement of the pulsed FIR lasers, the $25 \mathrm{~T}$ magnet, and the heterodyne-detection system. 
$10 \mathrm{~dB}$, since the polarization is only partly conserved in the circular oversized waveguides. This protection scheme proved to be adequate even though occasionally we had to make a new Schottky contact. At present the noise temperature of the detection system is $60000 \mathrm{~K}$; however, since the best system noise temperatures for these Schottky diodes are reported to be $2000 \mathrm{~K}$ [16], an improvement of a factor of 30 is, in principle, possible.

To describe the capabilities and limitations of our $604 \mathrm{GHz}$ ESE spectrometer, we will briefly describe the results of measurements on the free radicals $\alpha, \gamma$-bisdiphenylene- $\beta$-phenylallyl 1:1 complex with benzene (BDPA) and 2,2,6,6-tetramethyl-1-piperidinyloxy (TEMPO), which were dispersed in polystyrene and deposited as a film on the back mirror of the cavity. These materials have been chosen because they can be produced in any desired concentration and with any desired number of spins.

The power of the FIR pulses was adjusted by optimizing the system on the echo amplitude. Since we worked up to the limit of the power level of our FIR system, we are not sure that the actual pulses are real $\pi$ and $\pi / 2$ pulses. In Fig. 2, we show a single echo sequence at high sensitivity (single shot), where we can distinguish up to four echoes instead of the usual single echo. The sample, $30 \mu \mathrm{g}$ of BDPA dissolved in $3 \mathrm{mg}$ polystyrene, has $3.6 \times 10^{16}$ spins, which, as they are oriented in one direction, lead to a magnetic field that becomes comparable in magnitude to the alternating magnetic field $B_{1}$. Multiple echoes in concentrated samples have already been observed in low frequency EPR [4] and in solid ${ }^{3} \mathrm{He}$ in NMR [17]. The inset of Fig. 2 shows the integrated echo intensity for a fixed delay as a function of the magnetic field, recorded with a sweep velocity of $1 \mathrm{G} / \mathrm{s}$ and a continuous averaging over four shots (repetition rate $6 \mathrm{~Hz}$ ). A clear resonance with a linewidth of $10 \mathrm{G}$ can be identified. The noise of the spectrum is mainly due to fluctuations of the magnetic field.

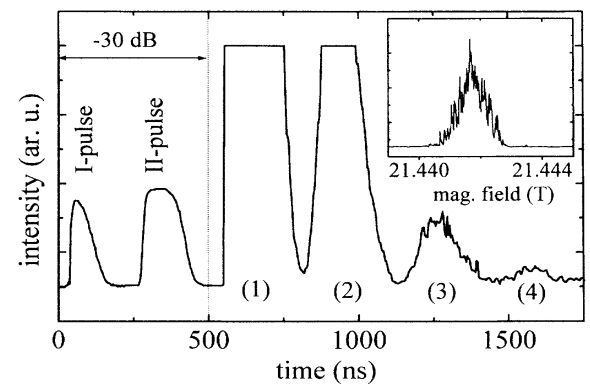

FIG. 2 A two-pulse echo sequence, for a BDPA sample at $5 \mathrm{~K}$, generating multiple echoes. The first two excitation pulses are attenuated by $30 \mathrm{~dB}$ with respect to the four echoes that follow. The inset shows the integrated echo intensity as a function of the magnetic field. The sweep velocity was $1 \mathrm{G} / \mathrm{s}$, and the signal was averaged continuously over four shots.
We also show here results of relaxation rate measurements on TEMPO $(0.17 \mathrm{mg})$ in $5 \mathrm{mg}$ polystyrene. The spin-memory decay rate can be determined by recording the echo height as a function of the delay time between the two excitation pulses. An exponential fit to the decay function yields a rate of $1.2 \times 10^{6} \mathrm{~s}^{-1}$ at $7 \mathrm{~K}$, more than 1 order of magnitude smaller than for the same sample at 9.5 GHz. The spin-memory decay rate at $9.5 \mathrm{GHz}$ shows no temperature dependence, whereas at $604 \mathrm{GHz}$ the decay rate increases with increasing temperature (Fig. 3). To understand the behavior of the spin-memory time, one has to look closer to the mechanism which is responsible for the loss of phase memory. It has been pointed out $[18,19]$ that the spin flip-flop process becomes important in relatively concentrated samples. In a spin flipflop process, one spin flips up and the other spin flips down, or vice versa, conserving the energy of the spin system. At $604 \mathrm{GHz}$ and low temperatures, the upper Zeeman level is almost empty, leading to only a few possibilities for flip-flop processes and consequently a small decay rate. We can describe the phase relaxation in a quantitative way assuming that the flip-flop rate is dependent only on the number of pairs that can undergo a flipflop process. This number is proportional to the product of the occupation of the upper and lower Zeeman levels, and we can use the following fit function: $r_{M}=a /\{[1+$ $\exp (b / T)][1+\exp (-b / T)]\}+r_{B}$ with parameters $a$ and $b$ and a parameter $r_{B}$ which describes a background rate which does not depend on the temperature. We obtain for the fit parameters $a=3.4 \times 10^{7} \mathrm{~s}^{-1}, b=(31 \pm 3) \mathrm{K}$ and $r_{B}=5.5 \times 10^{5} \mathrm{~s}^{-1}$. The obtained value for $b$ of $31 \mathrm{~K}$ is very close to the actual Zeeman temperature of $29 \mathrm{~K}$ $\left(\Theta_{Z}=\Delta E / k_{B}\right)$, justifying the chosen fit function. Furthermore, if we keep the parameters $a$ and $r_{B}$ and use for $b$ the Zeeman temperature at $9.5 \mathrm{GHz}$, we can describe reasonably well the behavior of the low frequency data.

The spin-lattice relaxation time $T_{1}$ can be determined in a saturation recovery experiment, applying a strong

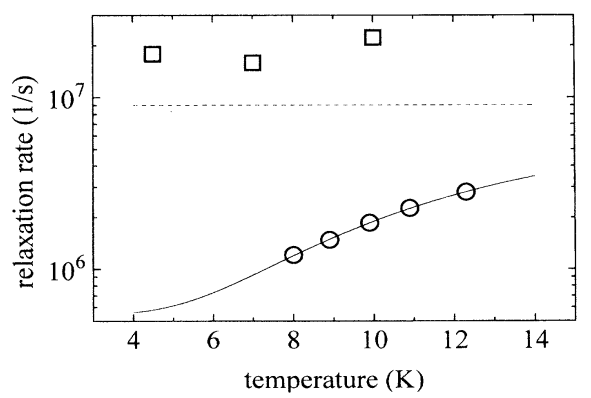

FIG. 3. Temperature dependence of the measured spinmemory decay rate $1 / T_{M}$. At $604 \mathrm{GHz}$ the spin-memory time (circles) depends strongly on the temperature. The spin-memory time, measured at $9.5 \mathrm{GHz}$ (squares), shows no temperature dependence. The solid and the dashed lines are fits for the 604 and $9.5 \mathrm{GHz}$ data, respectively (see text). 
first pulse to change the steady state population of the spin levels and by observing the time dependent return to the equilibrium value with the help of an echo sequence. This sequence is simpler to realize experimentally than the stimulated echo sequence, because the saturation recovery technique is less sensitive to whether the pulses are real $\pi / 2$ pulses than the stimulated echo sequence. In the inset of Fig. 4, it can be seen that the echo amplitude as a function of the delay time $\tau_{1}$ between the first pulse and the echo-pulse sequence increases with increasing delay time. An exponential fit yields a spin-lattice relaxation time of $15 \mu \mathrm{s}$, more than 1 order of magnitude longer than $T_{M}$. Therefore we can conclude that the measured $T_{M}$ times are due to spin-spin type processes and are not limited by spin-lattice relaxation.

An analysis of the sensitivity of our setup has shown that at present it is able to detect $5 \times 10^{12}$ spins in a single-shot experiment. However, we expect that this sensitivity can be increased by several orders of magnitude by improvements in the detection scheme and more sophisticated light guiding.

In conclusion, we have observed for the first time electron-spin echo at $604 \mathrm{GHz}$ in a magnetic field of $21.5 \mathrm{~T}$. The spectrometer, using a pulsed FIR laser system and quasioptical techniques, is not limited to one frequency, but can be extended to cover the frequency range from $245 \mathrm{GHz}$ up to the $\mathrm{THz}$ region. Although the spectrometer at the moment does not have the possibilities

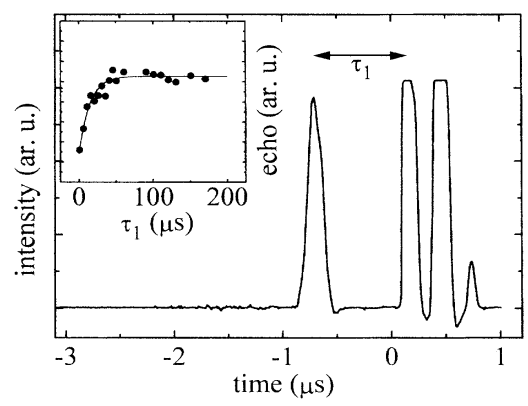

FIG. 4. A three-pulse sequence to measure the spin-lattice relaxation time. In the inset, the echo height is drawn as a function of the delay time $\tau_{1}$ between the first pulse (saturation pulse) and the echo-pulse sequence. From the exponential fit we find a spin-lattice relaxation time of $15 \mu \mathrm{s}$. and sensitivity as the spectrometers working at $95 \mathrm{GHz}$, it shows that ESE experiments can be performed at much higher frequencies, and first experimental results lead the way to new physical insights.

We would like to thank Dr. H.P. Röser for his help with the detection system, Dr. P. Petit for helping us to perform test measurements at $9 \mathrm{GHz}$, Dr. L.C. Brunel for many fruitful discussions, and Dr. F. Keilmann for lending us the second pulsed $\mathrm{CO}_{2}$ laser.

[1] E. L. Hahn, Phys. Rev. 80, 580 (1950).

[2] E. L. Hahn and D.E. Maxwell, Phys. Rev. 88, 1070 (1952).

[3] R. V. Blume, Phys. Rev. 109, 1867 (1958).

[4] J.P. Gordon and K. D. Bowers, Phys. Rev. Lett. 1, 368 (1958).

[5] D. E. Kaplan, M.E. Browne, and J. A. Cowen, Rev. Sci. Instrum. 32, 1182 (1961).

[6] W. B. Mims, K. Nassau, and J.D. Mc Gee, Phys. Rev. 123, 2059 (1961).

[7] R. Weber, J. A. J. M. Disselhorst, L. J. Prevo, J. Schmidt, and W. T. Wenckebach, J. Magn. Reson. 81, 129 (1989).

[8] T. Prisner (private communication).

[9] R. G. Griffin (private communication).

[10] Ya.S. Lebedev, in Modern Pulsed and Continuous-Wave Electron Spin Resonance, edited by L. Kevan and M. K. Bowman (Wiley, New York, 1990).

[11] W. B. Lynch, K. A. Earle, and J. H. Freed, Rev. Sci. Instrum. 59, 1345 (1988).

[12] F. Müller, M. A. Hopkins, N. Coron, M. Grynberg, L. C. Brunel, and G. Martinez, Rev. Sci. Instrum. 60, 3681 (1989).

[13] T. Y. Chang and T.J. Bridges, Opt. Commun. 1, 423 (1970).

[14] H. J. Schneider-Muntau, IEEE Trans. Magn. 17, 1775 (1981).

[15] R. Titz, B. Auel, W. Esch, H. P. Röser, and G. Schwaab, Infrared Phys. 30, 435 (1990).

[16] H. P. Röser (private communication).

[17] G. Deville, M. Bernier, and J. M. Delrieux, Phys. Rev. B 19, 5666 (1979).

[18] J. R. Klauder and P.W. Anderson, Phys. Rev. 125, 912 (1962).

[19] W. B. Mims, in Electron Paramagnetic Resonance, edited by S. Geschwind (Plenum, New York, 1972). 